\title{
AVALIAÇÃO DE METODOLOGIA PARA DETECÇÃO DE FUNGOS EM SEMENTES DE AMENDOIM $^{1}$
}

\author{
ANTONIO EDILSONDASILVA ARAÚJO², ANAPAULAGOMES DE CASTRO ${ }^{3}$, CLAUDIA ANTONIAVIEIRAROSSETTO $^{4}$
}

\begin{abstract}
RESUMO - O objetivo foi avaliar o efeito dos procedimentos de incubação e de desinfestação usando hipoclorito de sódio no desenvolvimento de fungos em teste de sanidade de sementes de amendoim (Arachis hypogaea L.). Foram realizados dois experimentos com dois lotes da cultivar Botutatu. No primeiro experimento, as sementes foram divididas em duas amostras, sendo uma imersa previamente em hipoclorito de sódio. A incubação destas foi realizada em meio agarizado (BDA, CZ) e em folhas de papel (sobre e em rolo), com ou sem restrição hídrica. No segundo experimento, as sementes foram imersas em hipoclorito de sódio a 1, 2, 35 e 10\%, por um, três, cinco e dez minutos e, posteriormente, foram incubadas em BDA. Os métodos de incubação em meio agarizado com restrição hídrica propiciaram maior recuperação de Aspergillus spp e de Cladosporium spp. nas sementes de amendoim sem desinfestação prévia. Houve menor ocorrência de Rhizopus spp. e Aspergillus niger nas sementes desinfestadas, independente do período de embebição e da concentração de hipoclorito de sódio.
\end{abstract}

Termos para indexação: Arachis hypogaea L., hipoclorito de sódio, restrição hídrica, Aspergillus spp..

\section{SANITARY QUALITY EVALUATIONAND MOLD GROWTH ON PEANUT SEEDS}

\begin{abstract}
The objective was to evaluate the effect of desinfection using sodium hypochloride and incubation procedures on the mold growth in the health test of peanut (Arachis hypogaea L.) seeds. Two experiments were carried out with two lots of cv. Botutatu. In the first experiment, the seeds were divided into two samples and one was submitted to immersion in sodium hypochloride. The incubation was realized in agar medium (PDA, CZ) and paper towel (blotter test and rolled paper), with or without water restriction. In the second experiment, the seeds were submitted to immersion in sodium hypochloride at 1,2,3,5 and 10\%, for one, three, five and ten minutes and they were incubated in PDA. The incubation methods in agar medium with water restriction promoted the highest recovery of Aspergillus spp. and Cladosporium spp. in the peanut seeds that had not been submitted to previous desinfection. Thef Rhizopus spp. and group Aspergillus niger incidence was lower in the disinfected seeds, regardless of the immersion period or sodium hypochlorite concentration.
\end{abstract}

Index terms: Arachis hypogaea L., sodium hypochloride, water restriction, Aspergillus spp.

\footnotetext{
${ }^{1}$ Submetido em 28/10/2003. Aceito para publicação em 20/05/2004

2 Eng. Agrônomo, Mestrando em Fitotecnia da UFRRJ. Email: edilson@ufrrj.br, Bolsista do CNPq.

${ }^{3}$ Graduanda em Agronomia da UFRRJ. Email: ana.castro21@bol.com.br,
}

Bolsista da FAPERJ.

${ }^{4}$ Eng. Agrônomo, Dr., Professora do Departamento de Fitotecnia da UFRRJ, Cx. Postal 74511, Rio de Janeiro, RJ, CEP 23.890-000. Email:cavrosse@ufrrj.br. Bolsista do CNPq. 


\section{INTRODUÇÃO}

Para o estabelecimento da cultura do amendoim é fundamental a avaliação da qualidade sanitária das sementes desta espécie, conjuntamente com a análise da germinação, principalmente quando se constata sementes mortas e plântulas anormais neste teste. Em sementes de amendoim, são freqüentemente detectados alguns fungos dos gêneros Aspergillus, Penicillium, Rhizopus, Fusarium (Ito et al., 1992) e Alternaria, Nigrospora, Trichoderma, Dothiorella e Pestalotia (Mariotto et al., 1982). Os fungos de maior incidência são Aspergillus spp. e Penicillium spp, que tem sido associados às sementes de baixa qualidade fisiológica (Mariotto et al., 1982). Espécies de Aspergillus dos grupos flavus e niger tem causado decréscimo de germinação (Harmon \& Pfleger, 1974; McLean et al., 1984) e, assim como Penicillium spp., tem promovido lesões nas plântulas, levando ao menor desenvolvimento destas (Backman \& Hammond, 1976, Ito et al., 1992). Estes fungos podem contaminar e infectar as sementes de amendoim no campo (Rossetto et al., 2003) e após a colheita (Ito et al., 1992). Em sementes de amendoim, Usberti \& Amaral (1999) constataram que para a invasão destes fungos, os teores de água das sementes devem ser maiores que 7\% (base úmida).

Para a avaliação da qualidade sanitária, métodos diversos têm sido empregados, visando a detecção de fungos associados às sementes de amendoim. Dentre eles, destaca-se o método do papel de filtro. Segundo Reis et al. (1999), muitos fungos contaminantes crescem rapidamente neste substrato, tais como, Aspergillus spp. e Rhizopus spp., podendo dificultar a identificação e a quantificação de fungos de crescimento lento, de tal forma que a incidência pode ser subestimada. Outro método é o de plaqueamento das sementes em meio de cultura agarizado, tais como, BDA (extrato de batata-dextrose-ágar) e CZ (czapeck) (Ito et al., 1992). Para Lucca Filho (1987), este é utilizado quando as condições de outros métodos, como a incubação em papel de filtro, não são adequadas para o crescimento vegetativo e esporulação de fungos e para a detecção de patógenos que produzem colônias características em meio de cultura. No entanto, este método pode ter sua prática prejudicada na detecção de patógenos, quando ocorre a germinação das sementes, dificultando a identificação dos fungos associados às mesmas (Coutinho et al., 2001).

Para inibir a germinação, quando as sementes são avaliadas pelos métodos de incubação em papel de filtro e em meio de cultura agarizado, tem sido utilizado 2,4diclorofenoxiacetato de sódio, assim como a técnica da restrição hídrica, induzida pela adição ao meio de cloreto de sódio, cloreto de potássio ou manitol (Braccini \& Dhingra, 1996; Coutinho et al., 2001). Estes solutos interferem na germinação tanto pelo efeito osmótico (Heydecker et al., 1995), quanto pela penetração de níveis tóxicos nas células (Braccini et al., 1996) e inibição da síntese e, ou, das atividades de enzimas hidrolíticas necessárias à germinação das sementes, provocada por concentração elevada de sais (Campos \& Assunção, 1990). Além disso, segundo Coutinho et al. (2001), a técnica de restrição hídrica minimiza as contaminações secundárias resultante de crescimento fúngico sobre os nutrientes exsudados das sementes mortas que são drenados pelo papel de filtro. Comparando os métodos de incubação, Ito et al. (1992) mostraram que o meio BDA acrescido de $6 \%$ de cloreto de sódio foi o que proporcionou maior recuperação de Aspergillus spp. e de Penicillium spp. em sementes de amendoim, que não foram previamente tratadas com hipoclorito de sódio, em relação ao método do papel de filtro. Já em sementes de soja, Pereira et al. (1994) detectaram, pelo método de papel de filtro com 2,4 diclorofenixiacetato de potássio, sem tratamento prévio das sementes, maior recuperação de Aspergillus spp. em relação ao método de incubação em meio ágar-salino, com desinfestação prévia das sementes.

Em relação ao tratamento prévio das sementes, tem sido recomendado em testes de sanidade de sementes de amendoim, o uso de hipoclorito de sódio $(\mathrm{NaOCl})$, na concentração de $1 \%$, por três minutos (Brasil, 1992). Este tratamento pode auxiliar na redução da contaminação das sementes (Coutinho et al., 2000; Zorato et al., 2001), facilitando a identificação de microrganismos nos tecidos internos das sementes (Hewett, 1979). Quanto à eliminação de microorganismos na superfície das sementes por este agente desinfestante, esta depende da espécie do fungo, da condição fisiológica da semente, da quantidade de contaminação superficial, do tempo de imersão, do $\mathrm{pH}$, da concentração do desinfetante, do agente químico a ser empregado (Zito et al., 1995; Sauer \& Burroughs, 1986). Para Zorato et al. (2001), a partir da concentração de 3\% de hipoclorito de sódio por cinco minutos, houve eliminação de A. flavus em sementes de soja. No entanto, em sementes de amendoim, o tratamento com hipoclorito propiciou redução da recuperação de Aspergillus spp., de Penicillium spp. e de Rhizopus spp., pelo método de incubação em BDA (Ito et al., 1992) e do papel de filtro (Prete, 1985 e Ito et al., 1992), embora tenha promovido menor ocorrência de Rhizopus spp..

Diante do exposto, o objetivo deste trabalho foi avaliar o efeito dos procedimentos de incubação e de desinfestação 
com hipoclorito de sódio no desenvolvimento de fungos em teste de sanidade de sementes de amendoim.

\section{MATERIAL E MÉTODOS}

Foram conduzidos dois experimentos no Laboratório de Análise de Sementes da UFRRJ com dois lotes de sementes de amendoim (Arachis hypogaea L.), cultivar Botutatu. Estes foram produzidos em Seropédica/RJ, no cultivo das águas (safra 2001/02) e no cultivo da seca (safra 2003), padronizados quanto ao tamanho em peneira de crivo oblongo (18/64"), armazenados em sacos de papel em câmara seca $\left(18 \pm 2^{\circ} \mathrm{C}\right.$ e $45 \pm 5 \%$ de UR do ar) por 150 e 30 dias e, denominados de lote 1 e 2, respectivamente.

No primeiro experimento, visando a avaliação dos métodos de incubação as sementes dos dois lotes foram divididas em duas amostras, sendo uma submetida ao tratamento prévio com hipoclorito de sódio, a $2 \%$, por cinco minutos e, em seguida, a lavagem em água destilada e a secagem sob fluxo laminar por uma hora. Posteriormente, as amostras foram colocadas em diferentes substratos, sendo cinco subamostras de 10 sementes em caixas plásticas tipo gerbox contendo três folhas de germibox, duas subamostras de 25 sementes em rolo de papel germitest e, cinco subamostras de 10 sementes em cada placa de Petri contendo meio agarizado (CZ - czapeck e BDA-batata, dextrose e ágar) acrescido de sulfato de estreptomicina (0,03\%). A estes substratos (meio de cultura agarizado e folhas de papel de filtro) foi adicionado ou não solução de $\mathrm{NaCl}$ (6\%), visando obter restrição hídrica e impedir a germinação das sementes, conforme descrito em Ito et al. (1992). A incubação foi realizada a $20^{\circ} \mathrm{C}$, por cinco dias, sob regime alternado de $12 \mathrm{~h}$ de luz (fornecida por quatro lâmpadas fluorescentes de 20W). Posteriormente, foi realizada a identificação e a quantificação dos fungos, com auxílio de microscópio estereoscópico. Quando necessário, foram feitas lâminas e usado o microscópio óptico. Foi determinada a porcentagem de cada fungo (Fusarium spp., Rhizopus spp., Cladosporium spp., Penicillium spp., Aspergillus spp.), com base em Singh (1992) e Silveira (1995). Para a caracterização dos isolados do grupo A. flavus, foi empregado o meio diferencial ADM (triptona, extrato de levedura e citrato férrico), conforme Bothast \& Fennell (1974). Cada isolado de Aspergillus spp. foi repicado para três placas de Petri contendo o referido meio. Após a incubação, a $25^{\circ} \mathrm{C}$, por $72 \mathrm{~h}$, sem luz, foi constatado que a presença de pigmentação laranja-amarela no verso da colônia indicou que o isolado pertencia ao grupo $A$. flavus.

No segundo experimento, visando a avaliação do tratamento prévio com hipoclorito de sódio, amostras de 50 sementes foram imersas em $130 \mathrm{ml}$ de hipoclorito de sódio a $1,2,3,5$ e $10 \%$, com respectivamente, $0,05,0,1,0,15,0,5$ e $1 \%$ de cloro ativo, por períodos de um, três, cinco e 10 minutos. Após a imersão, as sementes foram lavadas em água destilada e colocadas para secar sobre papel de filtro, sob fluxo laminar, por uma hora. Em seguida, foram realizados os testes de teor de água e de sanidade. Para o teste de teor de água, quatro subamostras de 15 sementes foram colocadas em estufa a $105 \pm 3{ }^{\circ} \mathrm{C}$, por 24 horas, de acordo com Brasil (1992). Para o de sanidade, cinco subamostras de 10 sementes foram incubadas a $20 \pm 2^{\circ} \mathrm{C}$, por cinco dias, sob regime alternado de $12 \mathrm{~h}$ de luz, em placas de Petri contendo meio BDA, acrescido de sulfato de estreptomicina $(0,03 \%)$ e de $\mathrm{NaCl}(6 \%)$, com base em Ito et al. (1992). Posteriormente, foi realizada a identificação e a quantificação dos fungos, de acordo com os procedimentos descritos anteriormente.

No primeiro experimento o delineamento experimental adotado foi inteiramente casualizado, em esquema fatorial (quatro métodos de incubação $\mathrm{x}$ dois procedimentos de restrição hídrica), com quatro repetições de 50 sementes. No segundo o delineamento experimental adotado foi inteiramente casualizado, em esquema fatorial (cinco concentrações de hipoclorito de sódio x quatro períodos de embebição), com três repetições.

A análise de variância foi realizada por lote e amostra (primeiro experimento) e por lote (segundo experimento), sendo que os dados em porcentagem foram transformados previamente em raiz quadrada de $(x+0,5)$. Nas Tabelas encontram-se os dados originais.

\section{RESULTADOS E DISCUSSÃO}

No primeiro experimento, quando não foi realizado o tratamento prévio com hipoclorito de sódio, foi verificado que houve efeito significativo da interação entre os métodos de incubação e os procedimentos de restrição hídrica na recuperação dos fungos dos gêneros Aspergillus, Penicillium e Cladosporium nas sementes do lote 1 e, dos gêneros Rhizopus e Fusarium nas sementes dos dois lotes (Tabelas 1 e 2). Os métodos de incubação em meio agarizado (BDA e $\mathrm{CZ}$ ), com restrição hídrica, propiciaram maior recuperação de fungos do grupo A. glaucus e dos gêneros Cladosporium e Aspergillus nas sementes do lote 1, em relação aos sem restrição hídrica (Tabela 1), assim como menor recuperação de Rhizopus spp. e de Fusarium spp. nas sementes dos dois 
TABELA 1. Incidência (\%) de fungos em sementes de amendoim, cv. Botutatu, do lote 1, incubadas em BDA, Czapek, papel de filtro, rolo de papel, com $(\mathrm{C} / \mathrm{R})$ ou sem $(\mathrm{S} / \mathrm{R})$ restrição hídrica, sem desinfestação com hipoclorito de sódio. Seropédica, RJ, 2002/2003.

\begin{tabular}{|c|c|c|c|c|c|c|c|c|c|c|c|}
\hline \multirow{2}{*}{ Tratamentos } & \multicolumn{3}{|c|}{ Rhizopus spp. } & \multicolumn{3}{|c|}{ Fusarium spp. } & \multicolumn{2}{|c|}{ Cladosporium spp. } & \multicolumn{3}{|c|}{ Penicillium spp. } \\
\hline & $\mathrm{C} / \mathrm{R}$ & $\mathrm{S} / \mathrm{R}$ & Média & $\mathrm{C} / \mathrm{R}$ & $\mathrm{S} / \mathrm{R}$ & Média & $\mathrm{S} / \mathrm{R}$ & Média & $\mathrm{C} / \mathrm{R}$ & $\mathrm{S} / \mathrm{R}$ & Média \\
\hline$\overline{\mathrm{BDA}}$ & $56,0 \mathrm{Ba}^{*}$ & $95,0 \mathrm{Aa}$ & 75,5 & $1,0 \mathrm{Ba}$ & $10,0 \mathrm{Ac}$ & 5,0 & $8,5 \mathrm{Aa} 0,5 \mathrm{Ba}$ & 4,5 & $43,5 \mathrm{Ab}$ & $29,0 \mathrm{Ab}$ & 36,3 \\
\hline Czapek & $3,5 \mathrm{Bc}$ & $62,5 \mathrm{Ab}$ & 33,0 & $0,0 \mathrm{Ba}$ & $22,5 \mathrm{Aa}$ & 11,3 & $4,5 \mathrm{Aa} 0,0 \mathrm{Ba}$ & 2,8 & $45,8 \mathrm{Ab}$ & $41,5 \mathrm{Aab}$ & 43,6 \\
\hline Papel de filtro & $12,5 \mathrm{Ac}$ & $14,5 \mathrm{Ac}$ & 13,5 & $4,0 \mathrm{Aa}$ & $6,5 \mathrm{Ab}$ & 5,2 & $0,5 \mathrm{Ab} 0,0 \mathrm{Aa}$ & 0,3 & $64,5 \mathrm{Aa}$ & $66,0 \mathrm{Aa}$ & 65,3 \\
\hline Rolo de papel & $20,0 \mathrm{Ab}$ & $21,0 \mathrm{Ac}$ & 20,5 & $0,5 \mathrm{Aa}$ & $0,5 \mathrm{Ac}$ & 0,5 & $0,0 \mathrm{Ab} \quad 0,5 \mathrm{Aa}$ & 0,3 & $82,5 \mathrm{Aa}$ & $25,5 \mathrm{Bb}$ & 54,0 \\
\hline Média & 20,5 & 48,3 & & 0,9 & 7,4 & & 3,4 & & 59,1 & 40,5 & \\
\hline C.V.(\%) & & 14,63 & & & 37,48 & & 38,48 & & & 16,77 & \\
\hline \multirow[t]{2}{*}{ Tratamentos } & \multicolumn{3}{|c|}{ Aspergillus spp. } & \multicolumn{3}{|c|}{ grupo Aspergillus flavus } & \multicolumn{2}{|c|}{$\begin{array}{c}\text { grupo Aspergillus } \\
\text { niger }\end{array}$} & \multicolumn{3}{|c|}{ grupo Aspergillus glaucus } \\
\hline & $\mathrm{C} / \mathrm{R}$ & $\mathrm{S} / \mathrm{R}$ & Média & $\mathrm{C} / \mathrm{R}$ & $\mathrm{S} / \mathrm{R}$ & $\overline{\text { Média }}$ & $\mathrm{S} / \mathrm{R}$ & Média & $\mathrm{C} / \mathrm{R}$ & $\mathrm{S} / \mathrm{R}$ & Média \\
\hline BDA & $91,5 \mathrm{Aa}$ & $19,0 \mathrm{Bb}$ & $\overline{55,3}$ & $25,5 \mathrm{Aa}$ & $6,5 \mathrm{Bb}$ & 16,0 & $4,5 \mathrm{Aa} 1,5 \mathrm{Bb}$ & 3,0 & $81,5 \mathrm{Aa}$ & $4,5 \mathrm{Bb}$ & 43,0 \\
\hline Czapek & $73,8 \mathrm{Aa}$ & $62,0 \mathrm{Ba}$ & 67,9 & $13,3 \mathrm{Bab}$ & $4,0 \mathrm{Aa}$ & 28,6 & $1,0 \mathrm{Ba} 1,5 \mathrm{Aa}$ & 1,2 & $66,3 \mathrm{Aa}$ & $13,0 \mathrm{Bb}$ & 39,0 \\
\hline Papel de filtro & $48,5 \mathrm{Ab}$ & $39,5 \mathrm{Ab}$ & 44,0 & $6,5 \mathrm{Ab}$ & $3,0 \mathrm{Ab}$ & 9,8 & $1,0 \mathrm{Aa} 1,0 \mathrm{Ab}$ & 1,0 & $24,5 \mathrm{Ab}$ & $19,5 \mathrm{Aa}$ & 22,0 \\
\hline Rolo de papel & $59,5 \mathrm{Ab}$ & $32,5 \mathrm{Ab}$ & 46,0 & $19,0 \mathrm{Aab}$ & $9,5 \mathrm{Ab}$ & 14,3 & $0,5 \mathrm{Aa} 0,5 \mathrm{Ab}$ & 0,5 & $34,5 \mathrm{Ab}$ & $21,5 \mathrm{Aa}$ & 28,0 \\
\hline Média & 68,3 & 38,3 & & 16,1 & 18,3 & & $1,7 \quad 5,1$ & & 68,3 & 38,3 & \\
\hline C.V.(\%) & & 15,95 & & & 26,14 & & 46,18 & & & 18,02 & \\
\hline
\end{tabular}

*Médias seguidas de mesma letra minúscula na coluna e maiúsculas na linha, não deferem entre si, pelo teste de Tukey a 5\% da probabilidade.

lotes (Tabelas 1 e 2). Os métodos de incubação em folhas de papel, com restrição hídrica, não diferiram dos sem a adição do soluto, na recuperação de fungos dos grupos $A$. flavus, A. glaucus e A. niger e dos gêneros Cladosporium e Aspergillus nas sementes do lote 1 (Tabela 1), e na de Rhizopus spp. e de Fusarium spp. nas sementes dos dois lotes (Tabelas 1 e 2). No entanto, para Coutinho et al. (2001), a técnica de restrição hídrica minimizou as contaminações secundárias resultante, principalmente, de crescimento de Rhizopus spp. sobre os nutrientes exsudados das sementes mortas que são drenados pelo papel de filtro.

Quando foi empregada a restrição hídrica, os métodos de incubação em meio agarizado (BDA e CZ) propiciaram menor recuperação de Penicillium spp., assim como maior recuperação de fungos do grupo $A$. glaucus e dos gêneros Cladosporium e Aspergillus nas sementes do lote 1, em relação aos métodos de incubação em folhas de papel (Tabela 1). Nas sementes do lote 2 , estes métodos também proporcionaram maior recuperação de Aspergillus spp. e de Cladosporium spp., porém, independentemente dos procedimentos de restrição hídrica (Tabela 2). Estes resultados estão de acordo com os obtidos por Ito et al. (1992), onde o método de plaqueamento em BDA acrescido de $6 \%$ de $\mathrm{NaCl}$, sem a desinfestação superficial com hipoclorito de sódio, foi o que propiciou maior recuperação de Aspergillus spp. e de
Penicillium spp., do que o do papel de filtro.

Neste experimento, não houve germinação das sementes nos substratos com restrição hídrica (dados não apresentados), pois a água contida nestes não foi suficiente para que as sementes atingissem a fase III do processo germinativo (Heydecker et al., 1975). Para Barba et al. (2002), a inibição da germinação das sementes induzida pela restrição hídrica do substrato favorece o maior contato desta com o substrato, uma vez que se evita o desenvolvimento da plúmula, estimulando a esporulação do fungo e facilitando a detecção e a quantificação destes.

Comparando a porcentagem de sementes dos dois lotes, contaminadas por fungos (Tabelas 1 e 2), foi constatada maior ocorrência de fungos dos gêneros Aspergillus, Cladosporium e Rhizopus nas do lote 1, que estavam armazenadas por maior período, embora apresentando $6,5 \%$ de água, valor abaixo do considerado favorável ao crescimento de fungos, com base em Usberti \& Amaral (1999). Também, Ito et al. (1992) detectaram estes fungos nas sementes desta espécie.

Quando foi realizado o tratamento prévio das sementes, não houve interação entre os métodos de incubação e os procedimentos de restrição hídrica na recuperação dos fungos dos gêneros Aspergillus, Rhizopus, Penicillium e Cladosporium nas sementes dos dois lotes (Tabelas 3 e 4). Assim, nas sementes dos dois lotes, a recuperação de fungos 
TABELA2. Incidência (\%) de fungos em sementes de amendoim, cv. Botutatu, do lote 2, incubadas em BDA, Czapek, papel de filtro, rolo de papel, com $(\mathrm{C} / \mathrm{R})$ ou sem $(\mathrm{S} / \mathrm{R})$ restrição hídrica, sem desinfestação com hipoclorito de sódio. Seropédica, RJ, 2002/2003.

\begin{tabular}{|c|c|c|c|c|c|c|c|c|c|}
\hline \multirow{2}{*}{ Tratamentos } & \multicolumn{3}{|c|}{ Rhizopus spp. } & \multicolumn{3}{|c|}{ Fusarium spp. } & \multicolumn{3}{|c|}{ Cladosporium spp. } \\
\hline & $\mathrm{C} / \mathrm{R}$ & $\mathrm{S} / \mathrm{R}$ & Média & $\mathrm{C} / \mathrm{R}$ & $\mathrm{S} / \mathrm{R}$ & Média & $\mathrm{C} / \mathrm{R}$ & $\mathrm{S} / \mathrm{R}$ & Média \\
\hline $\mathrm{BDA}$ & $0,00 \mathrm{Ba}^{*}$ & $10,00 \mathrm{Aa}$ & 5,00 & $24,00 \mathrm{Ba}$ & $41,00 \mathrm{Aa}$ & 32,50 & 2,00 & 3,00 & $2,50 \mathrm{a}$ \\
\hline Czapek & $0,00 \mathrm{Ba}$ & $9,00 \mathrm{Aa}$ & 4,50 & $27,00 \mathrm{Ba}$ & $47,50 \mathrm{Aa}$ & 37,25 & 0,00 & 1,50 & $0,75 \mathrm{ab}$ \\
\hline Papel de filtro & $0,00 \mathrm{Aa}$ & $0,50 \mathrm{Ab}$ & 0,25 & $15,50 \mathrm{Aa}$ & $24,50 \mathrm{Ab}$ & 20,00 & 0,00 & 1,00 & $0,50 \mathrm{~b}$ \\
\hline Rolo de papel & $2,00 \mathrm{Aa}$ & $1,50 \mathrm{Aab}$ & 1,75 & $17,00 \mathrm{Aa}$ & $14,00 \mathrm{Ab}$ & 15,50 & 0,00 & 0,00 & $0,00 \mathrm{~b}$ \\
\hline Média & 0,50 & 5,25 & & 20,87 & 31,75 & & $0,50 \mathrm{~A}$ & $1,38 \mathrm{~A}$ & \\
\hline C.V. $(\%)$ & & 53,97 & & & 15,87 & & & 51,49 & \\
\hline \multirow{2}{*}{ Tratamentos } & \multicolumn{3}{|c|}{ Penicillium spp. } & \multicolumn{3}{|c|}{ Aspergillus spp. } & \multicolumn{3}{|c|}{ grupo Aspergillus flavus } \\
\hline & $\mathrm{C} / \mathrm{R}$ & $\mathrm{S} / \mathrm{R}$ & Média & $\mathrm{C} / \mathrm{R}$ & $\mathrm{S} / \mathrm{R}$ & Média & $\mathrm{C} / \mathrm{R}$ & $\mathrm{S} / \mathrm{R}$ & Média \\
\hline BDA & 47,50 & 58,00 & $52,75 \mathrm{ab}$ & 0,00 & 0,50 & $0,25 \mathrm{ab}$ & 0,00 & 0,50 & $0,25 \mathrm{a}$ \\
\hline Czapek & 52,50 & 71,00 & $61,75 \mathrm{a}$ & 0,50 & 2,50 & $1,50 \mathrm{a}$ & 0,50 & 1,00 & $0,75 \mathrm{a}$ \\
\hline Papel de filtro & 27,00 & 41,50 & $34,25 b$ & 0,00 & 0,00 & $0,00 \mathrm{~b}$ & 0,00 & 0,00 & $0,00 \mathrm{a}$ \\
\hline Rolo de papel & 38,50 & 58,50 & $48,50 \mathrm{ab}$ & 0,00 & 0,00 & $0,00 \mathrm{~b}$ & 0,00 & 0,00 & $0,00 \mathrm{a}$ \\
\hline Média & $41,37 \mathrm{~B}$ & $57,25 \mathrm{~A}$ & & $0,30 \mathrm{~A}$ & $0,75 \mathrm{~A}$ & & $0,13 \mathrm{~A}$ & $0,38 \mathrm{~A}$ & \\
\hline C.V.(\%) & & 18,97 & & & 39,40 & & & 34,55 & \\
\hline
\end{tabular}

* Médias seguidas de mesma letra minúscula na coluna e maiúsculas na linha, não deferem entre si, pelo teste de Tukey a 5\% da probabilidade.

TABELA3. Incidência (\%) de fungos em sementes de amendoim, cv, Botutatu, do lote 1, incubadas em BDA, Czapek, papel de filtro, rolo de papel, com $(\mathrm{C} / \mathrm{R})$ ou sem $(\mathrm{S} / \mathrm{R})$ restrição hídrica, com desinfestação com hipoclorito de sódio, Seropédica, RJ, 2002/2003.

\begin{tabular}{|c|c|c|c|c|c|c|c|c|c|c|c|c|}
\hline \multirow[t]{2}{*}{ Tratamentos } & \multicolumn{3}{|c|}{ Rhizopus spp. } & \multicolumn{3}{|c|}{ Fusarium spp. } & \multicolumn{3}{|c|}{ Cladosporium spp. } & \multicolumn{3}{|c|}{ Penicillium spp. } \\
\hline & $\mathrm{C} / \mathrm{R}$ & $\mathrm{S} / \mathrm{R}$ & Média & $\mathrm{C} / \mathrm{R}$ & $\mathrm{S} / \mathrm{R}$ & Média & $\mathrm{C} / \mathrm{R}$ & $\mathrm{S} / \mathrm{R}$ & Média & $\mathrm{C} / \mathrm{R}$ & $\mathrm{S} / \mathrm{R}$ & Média \\
\hline $\mathrm{BDA}$ & 0,5 & 5,5 & $3,0 a^{*}$ & 12,5 & 21,0 & $16,8 \mathrm{a}$ & 1,5 & 1,0 & $1,3 a$ & 0,0 & 4,0 & $2,0 \mathrm{a}$ \\
\hline Czapek & 2,5 & 3,0 & $2,8 \mathrm{a}$ & 6,5 & 26,5 & $16,5 \mathrm{a}$ & 0,0 & 0,5 & $0,3 \mathrm{a}$ & 1,0 & 2,5 & $1,8 \mathrm{a}$ \\
\hline Papel de filtro & 0,0 & 2,5 & $1,3 \mathrm{a}$ & 13,5 & 9,0 & $11,3 \mathrm{a}$ & 0,5 & 0,0 & $0,3 \mathrm{a}$ & 1,5 & 2,0 & $1,8 \mathrm{a}$ \\
\hline Rolo de papel & 4,0 & 3,0 & $3,5^{\mathrm{a}}$ & 10,5 & 14,5 & $12,5 \mathrm{a}$ & 0,0 & 0,5 & $0,3 \mathrm{a}$ & 1,0 & 5,0 & $3,0 \mathrm{a}$ \\
\hline Média & $1,8 \mathrm{~A}$ & $3,5 \mathrm{~A}$ & & $10,8 \mathrm{~A}$ & $7,8 \mathrm{~A}$ & & $0,5 \mathrm{~A}$ & $0,5 \mathrm{~A}$ & & $0,9 \mathrm{~B}$ & $3,4 \mathrm{~A}$ & \\
\hline C.V.(\%) & & 2,55 & & & 27,38 & & & 35,80 & & & 49,74 & \\
\hline \multirow[t]{2}{*}{ Tratamentos } & \multicolumn{3}{|c|}{ Aspergillus spp. } & \multicolumn{6}{|c|}{ grupo Aspergillus flavus grupo Aspergillus niger } & \multicolumn{3}{|c|}{ grupo Aspergillus glaucus } \\
\hline & $\overline{\mathrm{C} / \mathrm{R}}$ & $\mathrm{S} / \mathrm{R}$ & Média & $\mathrm{C} / \mathrm{R}$ & $\mathrm{S} / \mathrm{R}$ & Média & $\mathrm{C} / \mathrm{R}$ & $\mathrm{S} / \mathrm{R}$ & Média & $\mathrm{C} / \mathrm{R}$ & $\mathrm{S} / \mathrm{R}$ & Média \\
\hline$\overline{\mathrm{BDA}}$ & 14,0 & 11,0 & $12,5 \mathrm{a}$ & 3,5 & 4,5 & $4,0 \mathrm{a}$ & 1,0 & 1,5 & $1,3 \mathrm{a}$ & 14,5 & 5,0 & $9,8 \mathrm{a}$ \\
\hline Czapek & 9,0 & 13,5 & $11,3 \mathrm{a}$ & 2,0 & 9,5 & $5,8 \mathrm{a}$ & 0,0 & 1,0 & $0,5 \mathrm{a}$ & 8,0 & 3,5 & $5,8 \mathrm{a}$ \\
\hline Papel de Filtro & 15,0 & 6,6 & $10,8 \mathrm{a}$ & 5,5 & 4,0 & $4,8 \mathrm{a}$ & 1,0 & 0,0 & $0,5 \mathrm{a}$ & 7,5 & 3,5 & $5,5 \mathrm{a}$ \\
\hline Rolo de papel & 14,0 & 5,0 & $9,5 \mathrm{a}$ & 2,0 & 1,0 & $1,5 \mathrm{a}$ & 4,0 & 0,5 & $2,3 \mathrm{a}$ & 5,2 & 6,4 & $5,8 \mathrm{a}$ \\
\hline Média & $3,0 \mathrm{~A}$ & $9,8 \mathrm{~A}$ & & $3,3 \mathrm{~A}$ & $4,8 \mathrm{~A}$ & & $1,5 \mathrm{~A}$ & $1,0 \mathrm{~A}$ & & $8,8 \mathrm{~A}$ & $4,6 \mathrm{~B}$ & \\
\hline 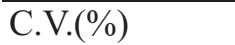 & & 37,37 & & & 50,08 & & & 60,75 & & & 33,75 & \\
\hline
\end{tabular}

* Médias seguidas de mesma letra minúscula na coluna e maiúsculas na linha, não deferem entre si, pelo teste de Tukey a 5\% da probabilidade.

do grupo A. flavus e dos gêneros Aspergillus, Rhizopus e Cladosporium não variou com os procedimentos de restrição hídrica e os métodos de incubação. Além disso, independente do método de incubação, houve menor recuperação de Penicillium spp., sob condição de restrição hídrica.
Comparando a porcentagem de sementes dos dois lotes contaminadas por fungos, após terem sido (Tabelas 3 e 4) ou não submetidas ao tratamento prévio (Tabelas 1 e 2), verificouse que a desinfestação das sementes propiciou menor ocorrência de Rhizopus spp., aumentando a recuperação de 
TABELA 4. Incidência (\%) de fungos em sementes de amendoim, cv, Botutatu, do lote 2, incubadas em BDA, Czapek, papel de filtro, rolo de papel, com $(\mathrm{C} / \mathrm{R})$ ou sem $(\mathrm{S} / \mathrm{R})$ restrição hídrica, com desinfestação com hipoclorito de sódio, Seropédica, RJ, 2002/2003.

\begin{tabular}{|c|c|c|c|c|c|c|c|c|c|}
\hline \multirow[t]{2}{*}{ Tratamentos } & \multicolumn{3}{|c|}{ Rhizopus spp. } & \multicolumn{3}{|c|}{ Fusarium spp. } & \multicolumn{3}{|c|}{ Cladosporium spp } \\
\hline & $\mathrm{C} / \mathrm{R}$ & $\mathrm{S} / \mathrm{R}$ & Média & $\mathrm{C} / \mathrm{R}$ & $\mathrm{S} / \mathrm{R}$ & Média & $\mathrm{C} / \mathrm{R}$ & $\mathrm{S} / \mathrm{R}$ & Média \\
\hline BDA & 0,00 & 5,00 & $2,50 \mathrm{a}^{*}$ & $9,00 \mathrm{Bab}$ & $33,50 \mathrm{Aa}$ & 21,25 & 0,50 & 0,00 & $0,25 \mathrm{a}$ \\
\hline Czapek & 0,50 & 0,50 & $0,50 \mathrm{a}$ & $17,00 \mathrm{Aa}$ & $23,00 \mathrm{Aab}$ & 20,25 & 0,00 & 0,50 & $0,25 \mathrm{a}$ \\
\hline Papel de filtro & 0,00 & 0,00 & $0,00 \mathrm{a}$ & $5,50 \mathrm{Ab}$ & $9,50 \mathrm{Ac}$ & 7,50 & 0,00 & 0,00 & $0,00 \mathrm{a}$ \\
\hline Rolo de papel & 0,00 & 1,50 & $0,75 \mathrm{a}$ & $19,00 \mathrm{Aa}$ & $11,00 \mathrm{Abc}$ & 15,00 & 0,00 & 0,00 & $0,00 \mathrm{a}$ \\
\hline Média & $0,13 \mathrm{~A}$ & $1,75 \mathrm{~A}$ & & 12,75 & 19,25 & & $0,13 \mathrm{~A}$ & $0,13 \mathrm{~A}$ & \\
\hline C.V. $(\%)$ & & 75,43 & & & 22,87 & & & 28,68 & \\
\hline \multirow[t]{2}{*}{ Tratamentos } & \multicolumn{3}{|c|}{ Penicillium spp. } & \multicolumn{3}{|c|}{ Aspergillus spp. } & \multicolumn{3}{|c|}{ grupo Aspergillus flavus } \\
\hline & $\mathrm{C} / \mathrm{R}$ & $\mathrm{S} / \mathrm{R}$ & Média & $\mathrm{C} / \mathrm{R}$ & $\mathrm{S} / \mathrm{R}$ & Média & $\mathrm{C} / \mathrm{R}$ & $\mathrm{S} / \mathrm{R}$ & Média \\
\hline BDA & 8,00 & 23,50 & $15,75 \mathrm{ab}$ & 3,50 & 2,00 & $2,75 a$ & 3,00 & 2,00 & $2,50 \mathrm{a}$ \\
\hline Czapek & 15,00 & 33,50 & $24,25 \mathrm{a}$ & 0,50 & 0,50 & $0,50 \mathrm{a}$ & 0,50 & 0,50 & $0,50 \mathrm{a}$ \\
\hline Papel de & 12,00 & 9,50 & $10,75 b$ & 0,00 & 0,50 & $0,25 \mathrm{a}$ & 0,00 & 0,00 & $0,00 \mathrm{a}$ \\
\hline Rolo de papel & 20,50 & 28,50 & $24,50 \mathrm{a}$ & 0,00 & 0,00 & $0,00 \mathrm{a}$ & 0,00 & 0,00 & $0,00 \mathrm{a}$ \\
\hline Média & $13,87 \mathrm{~B}$ & $23,75 \mathrm{~A}$ & & $1,00 \mathrm{~A}$ & $0,75 \mathrm{~A}$ & & $0,88 \mathrm{~A}$ & $0,63 \mathrm{~A}$ & \\
\hline C.V.(\%) & & 26,55 & & & 65,41 & & & 61,50 & \\
\hline
\end{tabular}

* Médias seguidas de mesma letra minúscula na coluna e maiúsculas na linha, não deferem entre si, pelo teste de Tukey a 5\% da probabilidade

Fusarium spp. e reduzindo a recuperação principalmente de fungos dos gêneros Aspergillus, Penicillium e Cladosporium. Também, Ito et al. (1992) constataram que a desinfestação das sementes de amendoim diminui a ocorrência de Rhizopus spp., porém reduz em até $90 \%$ a recuperação de Aspergillus spp. e de Penicillium spp. Assim, visando a detecção e a identificação de patógenos específicos, a decisão do emprego do tratamento prévio das sementes, bem como do método de incubação adequado, torna-se importante na análise da qualidade sanitária.

No segundo experimento, pode-se constatar pelas Tabelas 5 e 6, que houve efeito significativo da interação entre concentração de hipoclorito de sódio e período de embebição somente na ocorrência de Fusarium spp. nas sementes do lote 2. Assim, independente do período de embebição, com o emprego de hipoclorito de sódio, a 5 e $10 \%$, com 0,5 e 1,0\% de cloro ativo e $\mathrm{pH} 11,62$, foi constatado menor ocorrência de fungos do gênero Aspergillus, principalmente, os do grupo A. glaucus nas sementes do lote 1 (Tabela 5), assim como menor ocorrência de Penicillium spp. nas sementes do lote 2 (Tabela 6). Além disso, com o emprego destas concentrações, foi constatada eliminação de fungos dos grupos $A$. glaucus e A. niger nas sementes do lote 2 (Tabela 5). Para Zorato et al.
(2001), a partir da concentração de 3\% de hipoclorito de sódio, com $0,3 \%$ de cloro ativo ( $\mathrm{pH} 11,40$ ), por cinco minutos, houve decréscimo mas não eliminação de $A$. flavus em sementes de soja.

A partir de três minutos de imersão, independente da concentração de hipoclorito de sódio, foi verificado menor ocorrência de fungos do grupo A. flavus e dos gêneros Penicillium, Cladosporium e Aspergillus, nas sementes do lote 1 (Tabela 5) e de Penicillium spp. nas sementes do lote 2 (Tabela 6). Além disso, a partir deste período, foi constatada tendência de eliminação de Cladosporium spp. nas sementes do lote 2 .

Em relação ao Fusarium spp., foi verificada maior porcentagem de sementes do lote 1 contaminadas por estes, quando foram empregadas as concentrações de 5 e $10 \%$ de hipoclorito de sódio, independente do período de embebição, embora não tenha havido diferença significativa entre as demais concentrações (Tabela 5). Também, foi verificado maior porcentagem de sementes do lote 2 contaminadas por este fungo, quando foram usadas estas concentrações, por um minuto de exposição (Tabela 6). Por estes resultados, podese inferir que a menor ocorrência de Aspergillus spp., como constatada quando foram usadas estas concentrações de 
TABELA 5. Incidência (\%) de fungos em sementes de amendoim cv. Botutatu, do lote 1, com desinfestação com hipoclorito de sódio, a 1, 2, 3, 4, 5 e 10\%, por 1, 3, 5 e 10 minutos. Seropédica, RJ, 2002/2003.

\begin{tabular}{|c|c|c|c|c|c|c|c|c|c|c|}
\hline \multirow[t]{2}{*}{ Tratamentos } & \multicolumn{5}{|c|}{ Aspergillus flavus } & \multicolumn{5}{|c|}{ Aspergillus glaucus } \\
\hline & 1 & 3 & 5 & 10 & Média & 1 & 3 & 5 & 10 & Média \\
\hline $1 \%$ & 10,00 & 5,00 & 3,00 & 4,00 & $5,25 \mathrm{a}^{*}$ & 33,00 & 29,00 & 17,00 & 11,00 & $24,00 \mathrm{a}$ \\
\hline $2 \%$ & 4,00 & 2,00 & 0,00 & 1,00 & $1,50 \mathrm{a}$ & 18,00 & 19,00 & 15,00 & 8,00 & $16,75 \mathrm{a}$ \\
\hline $3 \%$ & 5,00 & 3,00 & 4,00 & 2,00 & $4,00 \mathrm{a}$ & 21,00 & 15,00 & 13,00 & 11,00 & $15,50 \mathrm{ab}$ \\
\hline $5 \%$ & 5,00 & 1,00 & 1,00 & 3,00 & $2,00 \mathrm{a}$ & 7,00 & 11,00 & 5,00 & 2,00 & $7,00 \mathrm{c}$ \\
\hline $10 \%$ & 3,00 & 2,00 & 1,00 & 1,00 & $1,75 \mathrm{a}$ & 16,00 & 14,00 & 4,00 & 2,00 & $9,50 \mathrm{bc}$ \\
\hline Média & $5,40 \mathrm{~A}$ & $2,60 \mathrm{~B}$ & $1,80 \mathrm{~B}$ & $2,20 \mathrm{~B}$ & & $19,00 \mathrm{~A}$ & $17,60 \mathrm{~A}$ & $10,80 \mathrm{~B}$ & $6,80 \mathrm{~B}$ & \\
\hline \multirow[t]{3}{*}{ C.V. $(\%)$} & \multicolumn{5}{|c|}{46,20} & \multicolumn{5}{|c|}{23,60} \\
\hline & \multicolumn{5}{|c|}{ Aspergillus niger } & \multicolumn{5}{|c|}{ Aspergillus spp. } \\
\hline & 1 & 3 & 5 & 10 & Média & 1 & 3 & 5 & 10 & Média \\
\hline$\overline{1 \%}$ & 2,00 & 1,00 & 1,00 & 1,00 & $1,25 \mathrm{a}$ & 45,00 & 34,00 & 21,00 & 17,00 & $30,25 a$ \\
\hline $2 \%$ & 3,00 & 1,00 & 1,00 & 1,00 & $1,50 \mathrm{a}$ & 25,00 & 22,00 & 16,00 & 10,00 & $19,75 \mathrm{a}$ \\
\hline $3 \%$ & 2,00 & 1,00 & 1,00 & 0,00 & $1,25 \mathrm{a}$ & 27,00 & 19,00 & 17,00 & 13,00 & $20,00 \mathrm{a}$ \\
\hline $5 \%$ & 1,00 & 2,00 & 0,00 & 1,00 & $0,75 \mathrm{a}$ & 13,00 & 13,00 & 6,00 & 6,00 & $9,50 \mathrm{~b}$ \\
\hline $10 \%$ & 1,00 & 0,00 & 0,00 & 0,00 & $0,25 \mathrm{a}$ & 20,00 & 16,00 & 5,00 & 3,00 & $11,50 \mathrm{~b}$ \\
\hline Média & $1,80 \mathrm{~A}$ & $1,00 \mathrm{~A}$ & $0,60 \mathrm{~A}$ & $0,60 \mathrm{~A}$ & & $26,00 \mathrm{~A}$ & $20,80 \mathrm{AB}$ & $13,00 \mathrm{BC}$ & $9,80 \mathrm{C}$ & \\
\hline \multirow[t]{3}{*}{ C.V.(\%) } & \multicolumn{5}{|c|}{53,43} & \multicolumn{5}{|c|}{24,74} \\
\hline & \multicolumn{5}{|c|}{ Penicillium spp. } & \multicolumn{5}{|c|}{ Rhizopus spp. } \\
\hline & 1 & 3 & 5 & 10 & Média & 1 & 3 & 5 & 10 & Média \\
\hline $1 \%$ & 9,00 & 3,00 & 3,00 & 1,00 & $4,50 \mathrm{a}$ & 0,00 & 1,00 & 1,00 & 0,00 & $0,75 \mathrm{a}$ \\
\hline $2 \%$ & 8,00 & 3,00 & 2,00 & 0,00 & $3,75 \mathrm{a}$ & 1,00 & 0,00 & 1,00 & 1,00 & $0,75 \mathrm{a}$ \\
\hline $3 \%$ & 6,00 & 0,00 & 1,00 & 2,00 & $2,00 \mathrm{a}$ & 0,00 & 1,00 & 1,00 & 0,00 & $0,75 \mathrm{a}$ \\
\hline $5 \%$ & 1,00 & 1,00 & 2,00 & 1,00 & $1,50 \mathrm{a}$ & 1,00 & 0,00 & 0,00 & 2,00 & $0,25 \mathrm{a}$ \\
\hline $10 \%$ & 3,00 & 3,00 & 1,00 & 0,00 & $2,00 \mathrm{a}$ & 0,00 & 2,00 & 0,00 & 0,00 & $0,50 \mathrm{a}$ \\
\hline Média & $5,40 \mathrm{~A}$ & $2,00 \mathrm{~B}$ & $1,80 \mathrm{~B}$ & $0,80 \mathrm{~B}$ & & $0,40 \mathrm{~A}$ & $0,80 \mathrm{~A}$ & $0,60 \mathrm{~A}$ & $0,60 \mathrm{~A}$ & \\
\hline \multirow{3}{*}{ C.V.(\%) } & \multicolumn{5}{|c|}{47,68} & \multicolumn{5}{|c|}{51,29} \\
\hline & \multicolumn{5}{|c|}{ Fusarium spp. } & \multicolumn{5}{|c|}{ Cladosporium spp. } \\
\hline & 1 & 3 & 5 & 10 & Média & 1 & 3 & 5 & 10 & Média \\
\hline $1 \%$ & 13,00 & 10,00 & 12,00 & 16,00 & $11,75 \mathrm{a}$ & 4,00 & 1,00 & 1,00 & 1,00 & $1,75 \mathrm{a}$ \\
\hline $2 \%$ & 23,00 & 5,00 & 12,00 & 9,00 & $13,00 \mathrm{a}$ & 3,00 & 3,00 & 0,00 & 1,00 & $1,50 \mathrm{a}$ \\
\hline $3 \%$ & 18,00 & 13,00 & 15,00 & 17,00 & $15,25 \mathrm{a}$ & 3,00 & 2,00 & 0,00 & 2,00 & $1,25 \mathrm{a}$ \\
\hline $5 \%$ & 22,00 & 19,00 & 17,00 & 27,00 & $18,75 \mathrm{a}$ & 3,00 & 1,00 & 1,00 & 1,00 & $1,50 \mathrm{a}$ \\
\hline $10 \%$ & 20,00 & 12,00 & 19,00 & 27,00 & $17,50 \mathrm{a}$ & 3,00 & 2,00 & 0,00 & 1,00 & $1,25 \mathrm{a}$ \\
\hline Média & $19,20 \mathrm{~A}$ & $11,80 \mathrm{~A}$ & $15,00 \mathrm{~A}$ & $19,20 \mathrm{~A}$ & & $3,20 \mathrm{~A}$ & $1,80 \mathrm{AB}$ & $0,40 \mathrm{~B}$ & $1,20 \mathrm{~B}$ & \\
\hline C.V.(\%) & \multicolumn{5}{|c|}{37,11} & \multicolumn{5}{|c|}{56,25} \\
\hline
\end{tabular}

* Médias seguidas de mesma letra minúscula na coluna e maiúsculas na linha, não deferem entre si, pelo teste de Tukey a 5\% da probabilidade

hipoclorito de sódio, pode ocultar outros patógenos nas sementes, tais como, os do gênero Fusarium, que são considerados causadores de redução da germinação de sementes (McLean et al., 1984; Faiad et al., 1997).

Pelas Tabelas 5 e 6, também foi verificado que, nas sementes dos dois lotes, não houve efeito da concentração de hipoclorito de sódio e do período de embebição na ocorrência de fungos do grupo $A$. niger e do gênero Rhizopus, que são freqüentes em sementes mortas (Phipps, 1984; Zorato et al., 2001), como também podem causar danos à qualidade fisiológica das sementes (Kabeere \& Taligoola, 1983; Novembre \& Marcos Filho, 1991). 
TABELA 6. Incidência (\%) de fungos em sementes de amendoim cv. Botutatu, do lote 2, com desinfestação com hipoclorito de sódio, a $1,2,3,4,5$ e $10 \%$, por $1,3,5$ e 10 minutos. Seropédica, RJ, 2002/2003.

\begin{tabular}{|c|c|c|c|c|c|c|c|c|c|c|}
\hline \multirow[t]{2}{*}{ Tratamentos } & \multicolumn{5}{|c|}{ Aspergillus flavus } & \multicolumn{5}{|c|}{ Aspergillus glaucus } \\
\hline & 1 & 3 & 5 & 10 & Média & 1 & 3 & 5 & 10 & Média \\
\hline $1 \%$ & 10,00 & 5,00 & 3,00 & 4,00 & $5,25 \mathrm{a}^{*}$ & 33,00 & 29,00 & 17,00 & 11,00 & $24,00 \mathrm{a}$ \\
\hline $2 \%$ & 4,00 & 2,00 & 0,00 & 1,00 & $1,50 \mathrm{a}$ & 18,00 & 19,00 & 15,00 & 8,00 & $16,75 \mathrm{a}$ \\
\hline $3 \%$ & 5,00 & 3,00 & 4,00 & 2,00 & $4,00 \mathrm{a}$ & 21,00 & 15,00 & 13,00 & 11,00 & $15,50 \mathrm{ab}$ \\
\hline $5 \%$ & 5,00 & 1,00 & 1,00 & 3,00 & $2,00 \mathrm{a}$ & 7,00 & 11,00 & 5,00 & 2,00 & $7,00 \mathrm{c}$ \\
\hline $10 \%$ & 3,00 & 2,00 & 1,00 & 1,00 & $1,75 \mathrm{a}$ & 16,00 & 14,00 & 4,00 & 2,00 & $9,50 \mathrm{bc}$ \\
\hline Média & $5,40 \mathrm{~A}$ & $2,60 \mathrm{~B}$ & $1,80 \mathrm{~B}$ & $2,20 \mathrm{~B}$ & & $19,00 \mathrm{~A}$ & $17,60 \mathrm{~A}$ & $10,80 \mathrm{~B}$ & $6,80 \mathrm{~B}$ & \\
\hline \multirow[t]{3}{*}{ C.V.(\%) } & \multicolumn{5}{|c|}{46,20} & \multicolumn{5}{|c|}{23,60} \\
\hline & \multicolumn{5}{|c|}{ Aspergillus niger } & \multicolumn{5}{|c|}{ Aspergillus spp. } \\
\hline & 1 & 3 & 5 & 10 & Média & 1 & 3 & 5 & 10 & Média \\
\hline $1 \%$ & 2,00 & 1,00 & 1,00 & 1,00 & $1,25 \mathrm{a}$ & 45,00 & 34,00 & 21,00 & 17,00 & $30,25 a$ \\
\hline $2 \%$ & 3,00 & 1,00 & 1,00 & 1,00 & $1,50 \mathrm{a}$ & 25,00 & 22,00 & 16,00 & 10,00 & $19,75 \mathrm{a}$ \\
\hline $3 \%$ & 2,00 & 1,00 & 1,00 & 0,00 & $1,25 \mathrm{a}$ & 27,00 & 19,00 & 17,00 & 13,00 & $20,00 \mathrm{a}$ \\
\hline $5 \%$ & 1,00 & 2,00 & 0,00 & 1,00 & $0,75 \mathrm{a}$ & 13,00 & 13,00 & 6,00 & 6,00 & $9,50 \mathrm{~b}$ \\
\hline $10 \%$ & 1,00 & 0,00 & 0,00 & 0,00 & $0,25 \mathrm{a}$ & 20,00 & 16,00 & 5,00 & 3,00 & $11,50 \mathrm{~b}$ \\
\hline Média & $1,80 \mathrm{~A}$ & $1,00 \mathrm{~A}$ & $0,60 \mathrm{~A}$ & $0,60 \mathrm{~A}$ & & $26,00 \mathrm{~A}$ & $20,80 \mathrm{AB}$ & $13,00 \mathrm{BC}$ & $9,80 \mathrm{C}$ & \\
\hline \multirow[t]{3}{*}{ C.V.(\%) } & \multicolumn{5}{|c|}{53,43} & \multicolumn{5}{|c|}{24,74} \\
\hline & \multicolumn{5}{|c|}{ Penicillium spp. } & \multicolumn{5}{|c|}{ Rhizopus spp. } \\
\hline & 1 & 3 & 5 & 10 & Média & 1 & 3 & 5 & 10 & Média \\
\hline $1 \%$ & 9,00 & 3,00 & 3,00 & 1,00 & $4,50 \mathrm{a}$ & 0,00 & 1,00 & 1,00 & 0,00 & $0,75 a$ \\
\hline $2 \%$ & 8,00 & 3,00 & 2,00 & 0,00 & $3,75 \mathrm{a}$ & 1,00 & 0,00 & 1,00 & 1,00 & $0,75 \mathrm{a}$ \\
\hline $3 \%$ & 6,00 & 0,00 & 1,00 & 2,00 & $2,00 \mathrm{a}$ & 0,00 & 1,00 & 1,00 & 0,00 & $0,75 \mathrm{a}$ \\
\hline $5 \%$ & 1,00 & 1,00 & 2,00 & 1,00 & $1,50 \mathrm{a}$ & 1,00 & 0,00 & 0,00 & 2,00 & $0,25 \mathrm{a}$ \\
\hline $10 \%$ & 3,00 & 3,00 & 1,00 & 0,00 & $2,00 \mathrm{a}$ & 0,00 & 2,00 & 0,00 & 0,00 & $0,50 \mathrm{a}$ \\
\hline Média & $5,40 \mathrm{~A}$ & $2,00 \mathrm{~B}$ & $1,80 \mathrm{~B}$ & $0,80 \mathrm{~B}$ & & $0,40 \mathrm{~A}$ & $0,80 \mathrm{~A}$ & $0,60 \mathrm{~A}$ & $0,60 \mathrm{~A}$ & \\
\hline \multirow[t]{3}{*}{ C.V.(\%) } & \multicolumn{5}{|c|}{47,68} & \multicolumn{5}{|c|}{51,29} \\
\hline & \multicolumn{5}{|c|}{ Fusarium spp. } & \multicolumn{5}{|c|}{ Cladosporium spp. } \\
\hline & 1 & 3 & 5 & 10 & Média & 1 & 3 & 5 & 10 & Média \\
\hline $1 \%$ & 13,00 & 10,00 & 12,00 & 16,00 & $11,75 \mathrm{a}$ & 4,00 & 1,00 & 1,00 & 1,00 & $1,75 \mathrm{a}$ \\
\hline $2 \%$ & 23,00 & 5,00 & 12,00 & 9,00 & $13,00 \mathrm{a}$ & 3,00 & 3,00 & 0,00 & 1,00 & $1,50 \mathrm{a}$ \\
\hline $3 \%$ & 18,00 & 13,00 & 15,00 & 17,00 & $15,25 \mathrm{a}$ & 3,00 & 2,00 & 0,00 & 2,00 & $1,25 \mathrm{a}$ \\
\hline $5 \%$ & 22,00 & 19,00 & 17,00 & 27,00 & $18,75 \mathrm{a}$ & 3,00 & 1,00 & 1,00 & 1,00 & $1,50 \mathrm{a}$ \\
\hline $10 \%$ & 20,00 & 12,00 & 19,00 & 27,00 & $17,50 \mathrm{a}$ & 3,00 & 2,00 & 0,00 & 1,00 & $1,25 \mathrm{a}$ \\
\hline Média & $19,20 \mathrm{~A}$ & $11,80 \mathrm{~A}$ & $15,00 \mathrm{~A}$ & $19,20 \mathrm{~A}$ & & $3,20 \mathrm{~A}$ & $1,80 \mathrm{AB}$ & $0,40 \mathrm{~B}$ & $1,20 \mathrm{~B}$ & \\
\hline C.V.(\%) & \multicolumn{5}{|c|}{37,11} & \multicolumn{5}{|c|}{56,25} \\
\hline
\end{tabular}

* Médias seguidas de mesma letra minúscula na coluna e maiúsculas na linha, não deferem entre si, pelo teste de Tukey a 5\% da probabilidade

\section{CONCLUSÕES}

Os métodos de incubação em meio agarizado com restrição hídrica propiciam maior recuperação de Aspergillus spp. e de Cladosporium spp., nas sementes de amendoim sem desinfestação prévia;

Com o emprego do tratamento prévio, há menor recuperação de Penicillium spp., Cladosporium spp. e Aspergillus spp. e de espécies do grupo A. flavus, independente dos procedimentos de incubação e de restrição hídrica;

Há menor ocorrência de Rhizopus spp. e Aspergillus niger nas sementes desinfestadas, independente do período de embebição e da concentração de hipoclorito de sódio 


\section{REFERÊNCIAS}

BACKMAN, P.A; HAMMOND, J.M. Germination losses associated with delayed application of seed treatment fungicides after peanut shelling. Plant Disease Reporter, Beltsville, v.60, n.1, p.1-3, 1976.

BARBA, J.T; REIS, E.M; FORCELINI, C.A. Comparação de métodos para detecção de Bipolaris sorokiniana em sementes de cevada. Fitopatologia Brasileira, Brasília, v.27, n.4, p.389-394, 2002.

BOTHAST, R.J.; FENNELL, D.I. A medium of rapid identification and enumeration of Aspergillus flavus and related organisms. Mycologia, Lawrence, v.66, n.3, p.365-369, 1974.

BRACCINI, A.L.; DHINGRA, O.D. Identificação de fungos em sementes de soja (Glycine max (L.) Merrill) e pepino (Cucumis sativus L.) por diferentes métodos de detecção. Revista Unimar, Marília, v.18, n.3, p. 495-503, 1996.

BRACCINI, A.L.; RUIZ, H.A; BRACCINI, M.C.L; REIS, M.S. Germinação e vigor de sementes de soja sob estresse hídrico induzido por solução de cloreto de sódio, manitol e polietilenoglicol. Revista Brasileira de Sementes, Brasília, v.18, n.1, p.10-16, 1996.

BRASIL. Ministério da Agricultura e Reforma Agrária. Regras para análise de sementes. Brasília: SNDA/DNDV/CLAV, 1992.365p.

CAMPOS, L.S; ASSUNÇÃO, M.V. Estresse salino e hídrico na germinação e vigor de sementes de arroz. Pesquisa Agropecuária Brasileira, Brasília, v.25, n.6, p.857-862, 1990.

COUTINHO, W.M.; PEREIRA. L.A.A; MACHADO, J.C; FREITAS-SILVA, O; PENA, R.C.M.; MAGALHÃES, F.H.L. Efeitos de hipoclorito de sódio na germinação de conídios de alguns fungos transmitidos por sementes. Fitopatologia Brasileira, Brasília, v.25, n.3, p.552-555, 2000.

COUTINHO, W.M.; MACHADO, J.C.; VIEIRA, M.G.G.C.; GUIMARÃES, R.M.; FERRERIRA, D.F. Uso da restrição hídrica na embebição ou retardamento da germinação de sementes de arroz e feijão submetidas ao teste de sanidade em meio ágar-água. Revista Brasileira de Sementes, Brasília, v.23, n.2, p.127-135, 2001.

FAIAD, M.G.R.; SALOMÃO, A.N.; CUNHA, R.D.; PADILHA, L.S. Efeito do hipoclorito de sódio sobre a qualidade fisiológica e sanitária de sementes de Commiphora lepthophloeos (Mart.) J.B. Gillet. Revista Brasileira de Sementes, Brasília, v.19, n.1, p.1417, 1997.

HARMON, G.G; PFLEGER, F.L. Pathogenicity and infection sites of Aspergillus species in stored seeds. Phytopathology, Saint Paul, v.64, n.10, p.1339-1344, 1974.

HEWETT, P.D. Pretreatment in seed health testing. 2. Duration of hypochlorite pre-treatment in the agar plate test for Ascochyta spp. Seed Science and Technology, Zürich, v.7, n. 1, p.83-85, 1979.

HEYDECKER, W; HIGGINS, J; TURNER, Y.J. Invigoration of seeds? Seed Science and Technology, Zürich, v.3, n.3/4, p.881$888,1975$.

ITO, M.F; BACCHI, L.M.A; MARINGONI, A.C; MENTEN, J.O.M. Comparação de métodos para detecção de Aspergillus spp. e
Penicillium spp. em sementes de amendoim (Arachis hypogaea L.). Summa Phytopathologica, Piracicaba, v. 18, n. 3, p. 262-268, 1992.

KABEERE, F; TALIGOOLA, H.K. Microflora and deterioration of soybean seeds in Uganda . Seed Science and Technology, Zürich, v.11, n.2, p.381-392, 1983.

LUCCA FILHO, O.A. Metodologia dos testes de sanidade de sementes. In: SOAVE, J.; WETZEL, M.M.V.S. (Ed.). Patologia de sementes. Campinas: Fundação Cargill, 1987. p.276-298.

MARIOTTO, P.R; SILVEIRA NETO, A.V.P; FIGUEIREDO, P; OLIVEIRA P.A; ARAÚJO, J.B.M. Efeito do tratamento de sementes de amendoim (Arachis hypogaea L.) com fungicidas. O Biológico, Campinas, v.48, n.3, p.56-60, 1982.

McLEAN, M.; DINI, M; BERJAK, K. Contribution to the characterization of the seed storage fungi: Aspergillus vesicolor and Aspergillus wentii. Seed Science and Technology, Zürich, v.12, n.2, p.437-446, 1984.

NOVEMBRE, A.D.L.C.; MARCOS FILHO, J. Tratamento com fungicida e conservação de sementes de feijão. Revista Brasileira de Sementes, Brasília, v.13, n.2, p.105-113, 1991.

PEREIRA, G.F.A.; MACHADO, J.C.; SILVA, R.L.X.; OLIVEIRA, S.M.A. Fungos de armazenamento em lotes de sementes de soja descartados no estado de Minas Gerais na safra 1989/90. Revista Brasileira de Sementes, Brasília, v.16, n.2, p. 216-219, 1994.

PHIPPS, P.M. Soybean and peanut seed treatment: new developments and needs. Plant Disease, Saint Paul, v.68, n.1, p.76-77, 1984.

PRETE, C. E.C. Escolha manual, seleção eletrônica pela cor, tratamento fungicida e qualidade de sementes de amendoim (Arachis hypogaea L.).1985. 73f. Dissertação (Mestrado em Agronomia) - Escola Superior de Agricultura Luiz de Queiroz, Universidade se São Paulo, Piracicaba, 1985.

REIS, E.M.; REIS, A.C; CASA, R.T; BLUM, M.M.C. Comparison of methods to detect leaf and head blighting fungi in small grain seeds. Summa Phytopathologica, Piracicaba, v.25, n.4, p.364-367, 1999.

ROSSETTO, C.A.V.; LIMA, T.M.; VIEGAS, E.C.; SILVA, F.O.; BITTENCOURT, A.M. Efeito da calagem, da colheita e da secagem na qualidade sanitária de amendoim na seca. Pesquisa Agropecuária Brasileira, Brasília, v.38, n.5, p. 567-573, 2003.

SAUER, D.B; BURROUGHS, Disinfection of seed surfaces with sodium hypochlorite. Phytopathology, Saint Paul, v.76, n.7, p.745$749,1986$.

SILVEIRA, V.D. Micologia. 5 ed. Rio de Janeiro: Editora Âmbito Cultural, 1995. 336p.

SINGH, K.; FRISVAD, J.C.; THRANE, U.; MATHUR, S.B. An illustrated manual on identification of some seed-borne Aspergilli, Fusaria, Penicillia, and their mycotoxins. Hellerup: Danish Government Institute of Seed Pathology for Developing Countries, 1992. 133p.

USBERTI, R.; AMARAL, H.M. Fungicide dressing timing, seed size, seed origin and fungal incidence effects on groundnut (Arachis hypogaea L.) storability Seed Science and Technology, Zürich, v.24, n.2, p.699-706, 1999. 
ZITO, R.K; SEDIYAMA, C. S; SEDIYAMA,T; GOMES, J.L.L; ROCHA, V.S. Hipoclorito de sódio e álcool na esterilização superficial de sementes de soja. Revista Ceres, Viçosa, v.42, n.244, p.637-643, 1995.
ZORATO, M.F.; HOMECHIN, M.; HENNING, A.A. Efeito da assepsia superficial com diferentes agentes químicos na incidência de microorganismos em sementes de soja. Revista Brasileira de Sementes, Brasília, v.23 n.1 p. 159-166, 2001.

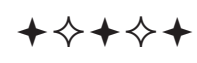

